\title{
Gaseous discs at intermediate redshifts from kinematic data modelling
}

\author{
R. Kipper ${ }^{1,2}$, A. Tamm ${ }^{1}$, P. Tenjes ${ }^{1,2}$ and E. Tempel ${ }^{1}$ \\ ${ }^{1}$ Tartu Observatory, Observatooriumi 1, 61602 Tõravere, Estonia \\ ${ }^{2}$ Institute of Physics, University of Tartu, 51010 Tartu, Estonia
}

\begin{abstract}
Our purpose is to measure thickness of gaseous discs in $0<z<1.2$ galaxies. As gas dispersions are sensitive to scale height of gaseous discs, we model the kinematics of galaxies using Jeans equations. The resulting thicknesses of gaseous discs at higher redshifts are more thicker (and arbitrary) while nearby ones are thinner. We also found that clumpiness of galaxy is a possible indicator of the gas disc thickness.
\end{abstract}

Keywords. galaxies: bulges - galaxies: kinematics and dynamics - galaxies: structure

\section{Introduction and methods}

Thickness of gaseous discs of galaxies is important to study in order to narrow down secular evolution and minor mergers: if we know how thin is gaseous disc, we know the initial thickness of stellar disc. Comparing it with observed thickness of disc it is possible to estimate the effect of secular evolution and possibly minor mergers.

We model the kinematics, measured by Weiner et al. (2006), of galaxies from GOODS$\mathrm{N}$ field, by estimating density distribution and using Jeans equations we calculate observable kinematics. Density estimation is based on superposition of four components: stellar disc, bulge, gaseous disc and dark matter halo. The distribution of stellar component comes from photometric modelling while its mass comes from spectral energy distribution fitting. The acquired mass is the sum of bulge and disc mass: the division between them was done manually. We assume that most of gaseous disc parameters are proportional to stellar ones, except mass, which comes from spectral energy distribution fitting and thickness, which is our aim to study and therefore free parameter.

\section{Results}

An example of our modelling results can be found in Fig. 1. Thickness of gaseous disc is difficult to measure, especially when galaxy is not edge-on. To overcome this difficulty we tried to find more simple estimate for that and our best finding is correlation with galaxy clumpiness. We calculated clumpiness using similar method by Conselice et al. (2003) where they subtracted blurred and observed image, summed the result and normalised with galaxy luminosity. Main difference compared with their approach is subtracting clumpiness of model image from observed one. Fig. 2 shows that this can only be rough estimator.

We also see from Fig. 2 that the scale height of gaseous disc is small in nearby galaxies while it has more arbitrary and bigger thicknesses at higher redshifts. This trend is compatible with nearby galaxies where thickness is estimated from dust distribution by Xilouris et al. (1999) and Bianchi (2007). Thinning of discs can be explained by "settling down" of gas and having fewer interactions between galaxies. 

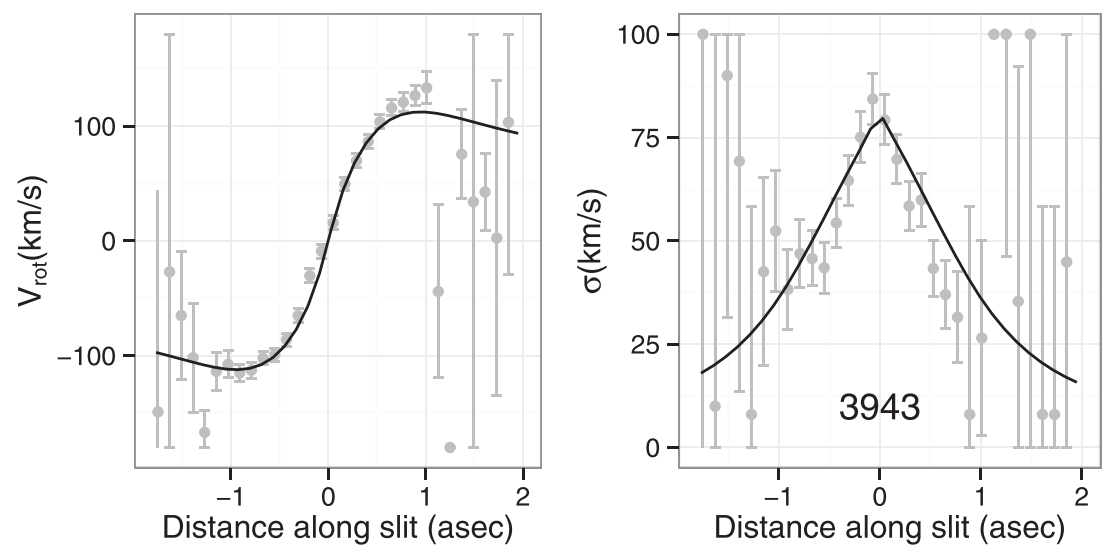

Figure 1. An example of kinematic modelling for galaxy TKRS 3943. Left panel shows rotation curve and right velocity dispersion distribution.
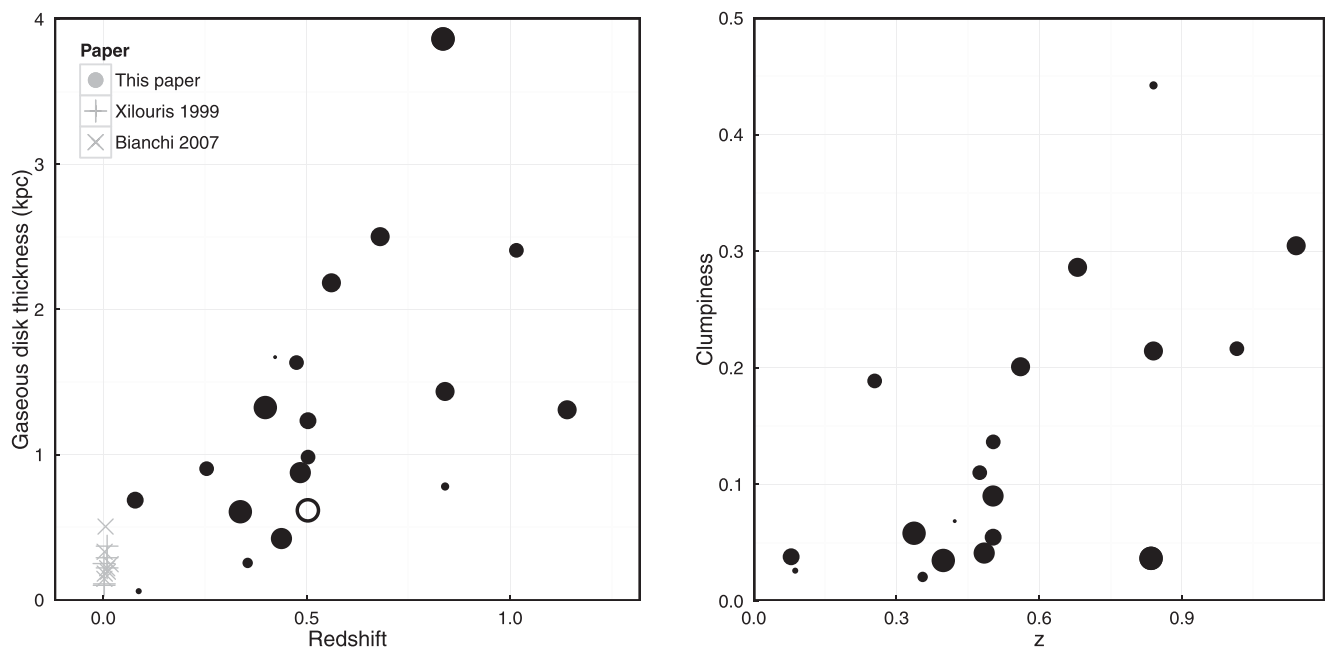

Figure 2. The dependence of the thickness of gaseous discs on redshift (left) and clumpiness (right). The size of points indicate the quality of our modelling.

\section{References}

Weiner, B. J., Willmer, C. N. A., Faber, S. M., Melbourne, J., Kassin, S. A., Phillips, A. C., Harker, J., Metevier, A. J., Bogt, N. P., \& Koo, D. C. 2006, A\&\&A, 653, 1027

Conselice, C. J. 2003, ApJS, 147, 1

Bianchi, S. 2007, A\& A, 471, 765

Xilouris, E. M., Byun, Y. I., Kylafis, N. D., Paleologou, E. V., \& Papamastorakis, J. 1999, A\&̊A, 344,868 